\title{
PRESTACIONES SOCIALES Y REPARTO JUSTO DE LA RIQUEZA
}

\section{Social benefits and fair distribution of wealth}

\author{
ÁNGEL ELÍAs* \\ Doctor en Derecho y decano de la Facultad de Relaciones Laborales y Trabajo Social \\ de la UPV/EHU
}

\section{RESUMEN}

Examino el Derecho Internacional, para analizar después las consecuencias de los grandes avances tecnológicos, que han permitido un enorme incremento de bienes y amenazan con la sustitución de muchos empleos. Sin embargo, constato la gran desigualdad imperante y sus nocivos efectos, por lo que es necesario que las riquezas y el tiempo del trabajo se repartan de forma justa, a lo que deben contribuir la renta básica y la reducción de la jornada laboral, así como un Observatorio para un reparto justo de la riqueza.

Palabras clave: riqueza, desigualdad, reparto, trabajo, renta básica.

\section{ABSTRACT}

I study the international law in order to make an analysis of the consequences carried out by big technological advances, that allowed a huge increase in goods and threaten many jobs. I also note the great existing inequality and its harmful effects, making necessary a fair distribution of wealth and time at work. This should contribute to a basic income and to a reduction of working hours, as well as to an observatory for a fair distribution of wealth.

Keywords: wealth, inequality, sharing, work, basic income.

* Correspondencia a: Ángel Elías. Facultad de Relaciones Laborales y Trabajo social (sede). B. ${ }^{\circ}$ Sarriena s/n. 48940 Leioa, Bizkaia-angel.elias@ehu.eus - https://orcid.org/0000-0002-7927-8835

Cómo citar: Elías, Ángel. (2018). "Prestaciones sociales y reparto justo de la riqueza»; Lan Harremanak, 40, 67-89 (https://doi.org/10.1387/lan-harremanak.20397).

Recibido: 19 noviembre, 2018; aceptado: 13 diciembre, 2018.

ISSN 1575-7048 - elSSN 2444-5819 / (C) 2018 UPV/EHU

(c) (i) Esta obra está bajo una licencia

Creative Commons Atribución 4.0 Internacional 


\section{SUMARIO}

1. Reconocimiento legal.-2. Hay medios apropiados para garantizar los anteriores derechos. 2.1. Medición de la riqueza y limitaciones del Producto Interior Bruto (PIB). 2.2. Nuevos conocimientos y tecnologías al servicio del progreso y el bienestar humano.-3. La otra cara de la moneda: una insoportable desigualdad.-4. Nuevas tecnologías y trabajo.-5. Necesidad de un reparto del trabajo.-6. Una nueva política de reparto.-7. Derecho a la renta básica universal e incondicional.-8. Propuesta de creación de un Observatorio para el reparto justo de la riqueza.-9. Bibliografía.

\section{Reconocimiento legal}

Sabemos que la Constitución es la norma de mayor jerarquía en España, habiendo sido aprobada por referéndum el 6 de diciembre de 1978, y publicada y vigente desde el 29 de diciembre de 1978. Su art. 10.2 establece que:

Las normas relativas a los derechos fundamentales y a las libertades que la Constitución reconoce se interpretarán de conformidad con la Declaración Universal de Derechos Humanos y los tratados y acuerdos internacionales sobre las mismas materias ratificados por España», lo que significa la automática vigencia de esa legislación internacional.

Y la Declaración Universal de Derechos Humanos de la ONU reconoce en el art. 25.1 que:

Toda persona tiene derecho a un nivel de vida adecuado que le asegure, así como a su familia, la salud y el bienestar, y en especial la alimentación, el vestido, la vivienda, la asistencia médica y los servicios sociales necesarios; tiene asimismo derecho a los seguros en caso de desempleo, enfermedad, invalidez, viudez, vejez u otros casos de pérdida de sus medios de subsistencia por circunstancias independientes de su voluntad.

La misma Declaración en el art. 22 ya había reconocido que:

Toda persona, como miembro de la sociedad, tiene derecho a la seguridad social, y a obtener, mediante el esfuerzo nacional y la cooperación internacional, habida cuenta de la organización y los recursos de cada Estado, la satisfacción de los 
derechos económicos, sociales y culturales, indispensables a su dignidad y al libre desarrollo de su personalidad.

Los anteriores derechos tienen su desarrollo en el art. 11.1 del Pacto Internacional de los Derechos Económicos, Sociales y Culturales de 1966, en lo sucesivo PIDESC, que forma parte del ordenamiento interno español tras haber sido ratificado por España (BOE, n. $\left.{ }^{\circ} 103,30-4-77\right)$, que reconoce

El derecho de toda persona a un nivel de vida adecuado para sí y su familia, incluso alimentación, vestido y vivienda adecuados, y a una mejora continua de las condiciones de existencia. ${ }^{1}$

\section{Y conforme al artículo 2.1 del PIDESC,}

Cada uno de los Estados Partes en el presente Pacto se compromete a adoptar medidas, tanto por separado como mediante la asistencia y la cooperación internacionales, especialmente económicas y técnicas, hasta el máximo de los recursos de que disponga, para lograr progresivamente, por todos los medios apropiados, inclusive en particular la adopción de medidas legislativas, la plena efectividad de los derechos aquí reconocidos.

La expresión "por todos los medios apropiados» debe ser interpretada en un sentido amplio, y dado que son los propios poderes públicos los que cuentan con la información relevante, les corresponde determinar qué medios son más apropiados para realizar progresivamente estos derechos, según el CESCR (1990). Esto significa que la administración pública debe asumir la carga de la prueba y demostrar que las medidas y políticas adoptadas son las idóneas para garantizar un adecuado nivel de cumplimiento de los derechos socioeconómicos. Asimismo, el carácter apropiado de un instrumento depende, en última instancia, de su eficacia para la materialización de los derechos sociales, según expresa en la Observación General 9.a , La aplicación interna del Pacto, el CESCR (1998). Por tanto, estamos ante unos derechos vinculantes para los Estados, que en el caso de Espańa incluye a los diferentes gobiernos autonómicos según el alcance de sus competencias y, además, permite la interposición de quejas individuales por vulneración de alguno de los derechos proclamados en el PIDESC, ya que España ratificó

1 A este respecto, el Comité Internacional de los Derechos Económicos, Sociales y Culturales, en lo sucesivo CESCR (2012) le recomendó a España: «Garantizar que todas las medidas de austeridad adoptadas identifiquen el contenido mínimo esencial de todos los derechos del Pacto, y tomar todas las medidas apropiadas para proteger este contenido esencial en cualquier circunstancia, especialmente para las personas y los grupos desfavorecidos y marginados.» 
en septiembre de 2010 el Protocolo Facultativo de dicho Pacto, en lo sucesivo (PF-PIDESC) ${ }^{2}$.

Más recientemente, el 25 de septiembre de 2015, los Estados miembros de Naciones Unidas acordaron en Nueva York los Objetivos de Desarrollo Sostenible, dentro de una amplia agenda de trabajo para la mejora de las condiciones de vida de las personas, con 17 Objetivos $^{3}$ y 169 metas interrelacionadas, de carácter integrado e indivisible. Una agenda, y en concreto, unos objetivos, los Objetivos de Desarrollo Sostenible (ODS), que retoman los anteriores Objetivos del Desarrollo del Milenio y pretenden conseguir lo que éstos no lograron, conjugando las tres dimensiones del desarrollo sostenible: económica, social y ambiental. El Gobierno español ha creado un Alto Comisionado para la Agenda

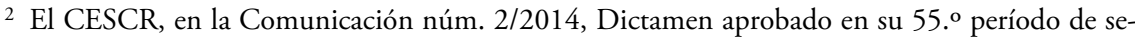
siones del 1 a 19 de junio de 2015, emitió una recomendación histórica por ser el primer caso decidido en virtud del PF-PIDESC, y determinar que España está violando el derecho a la vivienda. Grove, Chris and Ikawau, Daniela (2015) destacan las cinco razones por las que es importante esta Comunicación.

3 Son los siguientes:

- OSD 1. Poner fin a la pobreza en todas sus formas en todo el mundo.

- ODS 2. Poner fin al hambre, lograr la seguridad alimentaria y la mejora de la nutrición y promover la agricultura sostenible.

- ODS 3. Garantizar una vida sana y promover el bienestar para todos en todas las edades.

- ODS 4. Garantizar una educación inclusiva, equitativa y de calidad y promover oportunidades de aprendizaje durante toda la vida para todos.

- ODS 5. Lograr la igualdad entre los géneros y empoderar a las mujeres y a las niñas.

- ODS 6. Garantizar la disponibilidad de agua y su gestión sostenible y el saneamiento para todos.

- ODS 7. Garantizar el acceso a una energía asequible, segura, sostenible y moderna para todos.

—ODS 8. Promover el crecimiento económico sostenido, inclusivo y sostenible, el empleo pleno y productivo y el trabajo decente para todos.

—ODS 9. Construir infraestructuras resilientes, promover la industrialización inclusiva y sostenible y fomentar la innovación.

- ODS 10. Reducir la desigualdad en y entre los países.

- ODS 11. Lograr que las ciudades y los asentamientos humanos sean inclusivos, seguros, resilientes y sostenibles.

- ODS 12. Garantizar modalidades de consumo y producción sostenibles.

- ODS 13. Adoptar medidas urgentes para combatir el cambio climático y sus efectos.

- ODS 14. Conservar y utilizar en forma sostenible los océanos, los mares y los recursos marinos para el desarrollo sostenible.

- ODS 15. Proteger, restablecer y promover el uso sostenible de los ecosistemas terrestres, gestionar los bosques de forma sostenible, luchar contra la desertificación, detener e invertir la degradación de las tierras y poner freno a la pérdida de la diversidad biológica.

- ODS 16. Promover sociedades pacíficas e inclusivas para el desarrollo sostenible, facilitar el acceso a la justicia para todos y crear instituciones eficaces, responsables e inclusivas a todos los niveles.

—ODS 17. Fortalecer los medios de ejecución y revitalizar la Alianza Mundial para el Desarrollo Sostenible. 
2030, que dependerá directamente del Presidente del Gobierno y, según el Boletín Oficial del Estado (BOE) del 19 de junio de 2018, «se encargará de la coordinación de actuaciones para el cumplimiento» de los Objetivos de Naciones Unidas de Desarrollo Sostenible .

\section{Hay medios apropiados para garantizar los anteriores derechos}

Cuando entre nosotros se están planteando medidas de mejora de pensiones o de incremento en el salario mínimo interprofesional, llegan voces muy poderosas que afirman que es imposible, pues no hay riqueza que lo permita. Un buen ejemplo reciente es el artículo ¿Hacia una nueva reforma de las pensiones? Notas para el Pacto de Toledo, De la Fuente et al. $(2018)^{5}$, en cuyo resumen se analizan las posibles consecuencias de la derogación de la reforma de 2013 de la normativa sobre pensiones, y se dice:

Para evitar serios problemas de sostenibilidad a medio plazo, sería aconsejable que la reforma que ahora se está negociando en el Congreso contemple no sólo incrementos de ingresos sino también medidas de contención del gasto en pensiones que habrían de extenderse tanto a los pensionistas presentes como a los futuros. También es importante que el índice de actualización de las pensiones sea sensible a la situación financiera del sistema, aunque de forma menos drástica que el actual IRP.

Frente a esa visión que pone el acento en la insostenibilidad del gasto en pensiones o en salarios mínimos, es preciso tener en cuenta el avance exponencial de las nuevas tecnologías y la inteligencia artificial, con las consecuencias de creación de riqueza. Así lo hicimos un Consorcio de 21 socios de 11 países

4 Según el mismo BOE, sus funciones serán las siguientes:

- Realizar el seguimiento de las actuaciones de los órganos competentes de la Administración General del Estado para el cumplimiento de los objetivos de desarrollo sostenible y la Agenda 2030.

- Impulsar la elaboración y desarrollo de los planes y estrategias necesarios para el cumplimiento por España de la Agenda 2030.

- Evaluar, verificar y difundir el grado de avance en el cumplimiento de los objetivos de la Agenda 2030.

- Colaborar con el Ministerio de Asuntos Exteriores y Cooperación en la interlocución internacional de España en materia de implantación global de la Agenda 2030.

- Impulsar la elaboración de los sistemas de información y estadística necesarios para acreditar los avances en la consecución de los objetivos de la Agenda 2030.

5 Los autores de este informe participan en instituciones con indudable influencia mediática. Así, Angel de la Fuente en FEDEA e Instituto de Análisis Económico, CSIC, Miguel Ángel García Díaz en la Universidad Rey Juan Carlos y FEDEA, y Alfonso R. Sánchez en CUNEF y FEDEA. 
europeos que presentamos a la Convocatoria Horizon 2020 el 13 de marzo de 2018 el proyecto europeo denominado ToShare, Addressing technological transformations: exploring and sharing knowledge to co-create a Strategy for a diverse, inclusive, sustainable and smart Europe $e^{6}$, donde entendíamos que la revolución industrial debe generar no únicamente crecimiento económico, sino también bienestar social en un entorno de igualdad, justicia, equidad y sostenibilidad. El citado proyecto pretendía contribuir a la definición de un nuevo modelo económico y social coherente con el entorno de la cuarta revolución industrial, que incluiría:

— Una economía solidaria en un mundo sin fronteras ${ }^{7}$ : una economía que sirva a las necesidades de la comunidad y de cada una de las personas que la integran, prestando atención al papel de hombres y mujeres en las empresas y a los beneficios que obtienen de ella.

— La redistribución de la riqueza (fiscalidad, prioridades de gasto, protección social, renta básica universal): la riqueza obtenida debe repartirse de forma justa y debe permitir acceder a un sistema completo de protección social, que incluya la renta básica incondicional y universal, aportando seguridad y libertad a cada persona y favoreciendo la cohesión de la comunidad y la igualdad de género.

- En trabajo-empleo: reorganización y reparto del empleo y los trabajos (productivo, reproductivo y voluntario) para permitir el desarrollo de todas y cada una de las personas, asegurando la igualdad entre hombres y mujeres en su diversidad.

- Una educación inclusiva y permanente: actualmente hay una correlación inversa entre la velocidad en que se desarrollan las nuevas tecnologías y su impacto en la sociedad con el tiempo que necesitan las personas para adaptarse a los cambios. Se hace así necesario cuestionar el modelo

${ }^{6}$ Este proyecto SEP-210507853 se presentó en la call Socioeconomic and cultural transformations in the context of the fourth industrial revolution: Transformations-01-2018: Research for inclusive growth: addressing the socioeconomic effects of technological transformation $\mathrm{y}$, aunque no resultó aprobado, contó con la participación de importantes universidades, empresas e instituciones, así como el apoyo de otras más. Puede accederse al proyecto en el enlace https://www.ehu.eus/ documents/6902252/9450000/ToShare+PartB+section1-3+Final.pdf/ab304feb-39d7-f42f-fa1b-65b2164485da

7 Pero la élite de los países ricos apenas somos conscientes de nuestra suerte y mantenemos grandes prejuicios contra la apertura de fronteras, aunque solo el 3\% de la población mundial vive fuera de su país de nacimiento. En la historia la emigración ha sido la solución para combatir la pobreza y así lo proclama el artículo 13 de la Declaración Universal de los Derechos Humanos al reconocer que toda persona tiene derecho a dejar su país, aunque no garantiza el derecho a desplazarse a otras tierras. Reza así:

1. Toda persona tiene derecho a circular libremente y a elegir su residencia en el territorio de un Estado.

2. Toda persona tiene derecho a salir de cualquier país, incluso del propio, y a regresar a su país. 
educativo y de instrucción actual, precisando una educación a lo largo de toda la vida para asegurar personas con competencias y valores para ser felices y de cara a una evolución en el contenido y en la organización del trabajo.

Pero volviendo a la pregunta de si hay medios suficientes, un primer aspecto es su medición.

\subsection{Medición de la riqueza y limitaciones del Producto Interior Bruto (PIB)}

El deseo de medir la riqueza es muy antiguo y se remonta a la época de los fisiócratas, que creían que toda la riqueza salía de la tierra. Así, en 1665, el inglés Petty, William fue el primero en presentar un cálculo de lo que denominó renta nacional. Después, Adam, Smith, padre de la ciencia económica moderna, defendió que la riqueza se fundamentaba solo en la agricultura y la industria, pero no en el sector de servicios. Fue el economista Marshall Alfred, en el siglo XIX, el primero en argumentar que no es la naturaleza sino el precio de los productos lo que importa.

Pero, sin duda, es el PIB el concepto que desde mediados del siglo xx y gracias a kuznets, Simon, profesor ruso que lo inventó, se utiliza con mayor frecuencia como indicador de riqueza. Podemos definir el $\mathrm{PIB}^{8}$ como la suma de todos los bienes y servicios que produce un país, ajustado conforme a fluctuaciones estacionales, la inflación y el poder adquisitivo o, dicho de otra forma, el valor monetario de la producción de un territorio en un periodo de referencia, normalmente un año. Su cálculo conecta numerosos y diversos valores numéri$\cos ^{9} \mathrm{y}$ toma centenares de decisiones totalmente subjetivas sobre lo que incluir y descartar. Por ello, a continuación, analizo sus limitaciones.

Empezando por lo más básico, el PIB nada dice sobre la riqueza, ya que hay países más ricos aunque debido a su menor población su PIB sea menor, lo cual nos lleva a la renta per cápita, que es una media que no proporciona infor-

\footnotetext{
8 Siguiendo a Seyfferth, Ansgar (¿Cómo se mide la desigualdad?, 2014), el PIB mide la producción llevada a cabo en un país, independientemente de la residencia de quien la genera, a diferencia del producto nacional bruto (PNB) de un país que incluye los productos y servicios generados por sus residentes. En la mayoría de los países la diferencia entre ambos indicadores no es demasiado importante, pero sí en un país pequeño como Luxemburgo, donde casi la mitad de los trabajadores reside en los países vecinos, contribuyendo al PNB de los mismos, pero al PIB luxemburgués. De este modo inflan la renta per cápita luxemburguesa al entrar en el cálculo en el numerador (PIB) pero no en el denominador (población residente), o dicho de otro modo, todo el PIB del ducado, inclusive la parte que pertenece a los residentes extranjeros, es repartido en las estadísticas oficiales solo entre la población residente, que por tanto aparece como más rica de lo que realmente es.

9 Cuando Naciones Unidas publicó en 1953 sus primeras directrices estándar para calcular el PIB ocupó menos de 50 páginas; sin embargo, la edición más reciente del año 2008, tiene 722 páginas.
} 
mación sobre el reparto de la riqueza ${ }^{10}$. Para usar la renta per cápita como indicador del nivel de vida hay que ponerla también en relación con el nivel de pre$\operatorname{cios}^{11}$. Y para computar las diferencias de precios entre diferentes países se emplea el concepto de la paridad del poder adquisitivo ${ }^{12}$, lo que ajusta la renta per cápita a la baja en los países más caros y al alza en los países con un coste de vida menor, de modo que se pueden comparar los niveles de vida en términos de poder adquisitivo. Sin embargo, este ajuste se suele llevar a cabo solamente a nivel de país, a pesar de que los precios también pueden variar mucho dentro de cada país, un aspecto a tener en cuenta al comparar rentas per cápita regionales.

También quedan excluidas del PIB la economía sumergida, cuyo tamaño solo puede estimarse, y los servicios a la comunidad, los esfuerzos para no contaminar el aire, las aportaciones voluntarias no retribuidas que, en sus diversas modalidades de trabajo no remunerado, como el voluntario o el cuidado de personas y las tareas domésticas, representan más de la mitad de la totalidad del trabajo. Por ejemplo, si alguien se casa con quien le llevaba las tareas del hogar y el cuidado de sus hijas/os, dejando de pagarle con un salario a partir de entonces, el trabajo de esa persona ya no entra en el PIB aunque siga haciendo lo mismo que antes y tenga ahora un mayor nivel de vida.

Además, desastres naturales o guerras traen frecuentemente consigo el incremento del PIB, a pesar de que tal como recuerda Coyle, Diane (2014: 14), el mismo kuznets, Simon advirtió contra la inclusión en el mismo de los gastos de los sectores militar, publicitario y financiero.

Por otro lado, muchos servicios públicos se financian a través de los impuestos, sin que la ciudadanía pague al usarlos o solo abone una pequeña tasa que no cubre ni de lejos su coste, por lo que no hay precios de mercado con los que valorar la contribución de estos servicios al PIB. Se valoran simplemente por su coste, de forma que un incremento del gasto en estos servicios y, con ello, en el $\mathrm{PIB}$, puede ser fruto de la ineficacia y del despilfarro.

10 Una minoría muy rica puede subirla considerablemente mientras que la mayoría de la población se sitúa debajo de la media, tal como explica en su bloc Seyfferth, Ansgar (La omnipresente media estadística - ¿Qué nos dice y qué nos oculta?, 2014). Por ejemplo, un país con élites muy ricas y gran parte de la población viviendo en circunstancias muy modestas puede tener una renta per cápita parecida a la de un país igualitario, ya que los extremos se compensan de cara a la media, de forma que las enormes diferencias socioeconómicas entre ambas sociedades no quedan reflejadas en la renta per cápita. Para medirlas se requieren indicadores de desigualdad.

11 Para tener en cuenta su variación con el tiempo, normalmente al alza, es decir inflación, se divide el PIB o la renta per cápita nominal entre el llamado deflactor, que indica el nivel de precios relativo al de un año de referencia que se llama ańo base, para obtener así la evolución real, descontando el efecto de la inflación.

12 De esta forma la renta per cápita de un país se divide entre su índice de nivel de precios, lo que indica el nivel de precios del país relativo a un nivel de referencia que, en las estadísticas de la Unión Europea, es la media. 
Tampoco el PIB es eficaz para calcular los avances en conocimiento, pues los ordenadores, cámaras y teléfonos son más inteligentes, rápidos y vistosos que nunca, pero también más baratos y, por tanto, apenas aportan al PIB. Hace 30 años un GB costaba 300.000 euros y hoy vale menos de 10 centavos. bienestar.

En definitiva, el crecimiento en el PIB no puede equipararse al progreso y

\subsection{Nuevos conocimientos y tecnologías al servicio del progreso y el bienestar humano}

La innovación tecnológica, especialmente desde inicios de este siglo XXI con el acelerado desarrollo de internet, el sistema GPS, las pantallas táctiles, los dispositivos de grabación, los buscadores de algoritmos y un largo etcétera, está afectando de modo decisivo a nuestro estilo de vida. A modo de ejemplo, en el año 1965 los mejores ordenadores llevaban solo 30 transistores, mientras que en el año 2013 una consola tenía ya 5 mil millones de transistores; o en el año 1997 la computadora más rápida del mundo ASCI Red ofrecía una velocidad punta de un teraflop, ocupando una pista de tenis y costando 55 millones de dólares, mientras que en el año 2013 una Play Station 4 alcanzaba fácilmente 2 teraflops. Hoy sabemos que las nuevas generaciones de robots superan al hombre no solo en poder muscular, sino también en capacidad mental. Estos grandes avances tecnológicos han permitido que por primera vez en la historia haya suficiente riqueza para cubrir las necesidades que permitan una vida digna.

Como nos recuerda el historiador Bregman, Rutger (2016: 11 y ss.) en el año 1820 el 94\% de la población vivía en pobreza extrema, en el año 1981 el $44 \%$ y en el año 2012 el 10\%. Hoy en Holanda una persona sin techo tiene más dinero que el o la holandesa media del año 1954. No hubo grandes cambios en la historia hasta la década de 1880 , pero hoy la renta per cápita es 10 veces la del año 1850 y la o el italiano medio es 15 veces más rico que el del año 1880 . La economía global es 250 veces mayor que la de la Revolución Industrial. En el año 1800 todos los países eran pobres en riqueza y en salud, mientras que hoy incluso los países más pobres del África subsahariana están mejor que los más prósperos de entonces. La esperanza de vida ha pasado de 35 años en el año 1900 a 64 años en el año 1990 y a 70 años en el año 2012. El porcentaje de personas que tienen menos de 2000 calorías diarias ha bajado del $51 \%$ en el año $1965 \mathrm{al}$ 3\% en el año 2005. Entre los años 1990 y 2012, 2.100 millones de personas accedieron a agua potable, el número de niñas/os con retraso en el crecimiento disminuyó en una tercera parte, la mortalidad infantil descendió un $41 \%$ y el número de muertes de mujeres en el parto se redujo a la mitad. En el año 1962 el 41\% de las niñas y nińos no iban a la escuela y hoy el porcentaje es menor al $10 \%$. 


\section{La otra cara de la moneda: una insoportable desigualdad}

Pero este progreso viene acompañado de que la riqueza se encuentra concentrada en muy pocas manos. Los economistas observan que el número de ganadoras y ganadores cada vez es más pequeño, ya que ser un poco mejor que el resto significa ganar no solo la batalla, sino también la guerra, es decir, apropiarse de todo. La desigualdad se ha disparado en casi todos los países desarrollados y, por ejemplo, como señala Luque Víctor (2015: 88-89), la brecha entre personas ricas y pobres en Estados Unidos es mayor que la existente en la antigua Roma, donde la economía se basaba en el trabajo de personas esclavas. Somos una sociedad global en la que tan sólo 8 personas tienen tanto como la mitad del mundo, es decir, como 3.600 millones.

Los Informes anuales de Oxfam reflejan cómo la concentración de riqueza en el mundo se ha agudizado, amenazando la estabilidad y el crecimiento mundial. Así, en 2017 (Oxfam, 2018: 1), el número de personas cuyas fortunas superan los mil millones alcanzó su máximo histórico, con un nuevo milmillonario cada dos días. En este momento según la publicación Forbes hay 2.208 milmillonarios en todo el mundo que suman 9,1 trillones de dólares, y las diez personas con mayor fortuna son hombres (Kroll, Luisa y Dolan, Kerry, 2018). La riqueza de estos milmillonarios también experimentó un enorme crecimiento, lo suficiente como para poder terminar hasta siete veces con la extrema pobreza en el mundo. El $82 \%$ del crecimiento de la riqueza mundial durante el último ańo fue a parar a manos del $1 \%$ más rico, mientras que el del $50 \%$ más pobre de la población mundial no aumentó lo más mínimo. En 2017, Oxfam y Development Finance International elaboraron el primer índice para medir el grado de compromiso de los Gobiernos con la reducción de la brecha entre ricos y pobres, que es el $10 .^{\circ}$ de los Objetivos de Desarrollo Sostenible. El 9 de octubre de 2018 ha publicado la segunda edición, donde se muestra cómo algunos países tales como Corea del Sur, Namibia y Uruguay están dando grandes pasos para reducir la desigualdad, mientras países como la India o Nigeria reciben una puntuación muy baja. Entre los países ricos, también destaca, por ejemplo, Estados Unidos, como uno de los peor clasificados, lo que demuestra su falta de compromiso para reducir la desigualdad.

No obstante, la evolución de la desigualdad a nivel internacional está aumentando o reduciéndose en función de si se analiza dentro de los países o entre ellos. Así, según Firebaugh Glenn (2009), una de las tendencias principales de la desigualdad mundial daba muestras de estar invirtiéndose pasando de la creciente desigualdad entre países y la constante o menguante desigualdad dentro de los países, a la desigualdad menguante entre países y la creciente desigualdad dentro de ellos.

Sin embargo, las brechas de desigualdad entre gente que vive en el mismo país no son nada en comparación con las que separan a la ciudadanía del mundo 
(Bregman, Rutger, 2016: 199 a 211). Hoy, el 8\% más rico gana la mitad de los ingresos totales del mundo, y el $1 \%$ más rico posee más de la mitad de las riquezas. Solo las 62 personas más ricas de la tierra poseen lo mismo que la mitad más pobre del mundo. Los mil millones de personas más pobres solo suponen el $1 \%$ de todo el consumo, mientras que los mil millones más ricos el $72 \%$ del consumo. Hoy, una persona que vive en el umbral de pobreza en Estados Unidos pertenece al $14 \%$ más rico del mundo, y quien gana un salario medio pertenece al $4 \%$ más rico. Una niña somalí tiene un $20 \%$ de probabilidades de morir antes de cumplir los 5 años, mientras quienes luchaban en primera línea en la Guerra de Secesión tenían un índice de mortalidad del 6,7\%, del 1,8\% en la Segunda Guerra Mundial, y del 0,5\% en la guerra de Vietnam. Sin embargo, no dudamos en repatriar a esa niña si resulta que su madre no es una refugiada política que demuestre una abierta persecución por sus ideas o religión. Mientras en el siglo XIX la desigualdad era cuestión de clases, hoy es de ubicación. En los denominados países desarrollados el umbral de pobreza es 17 veces mayor que en las restantes. Un estadounidense por el mismo trabajo gana el triple que un/a boliviana de la misma edad, sexo, y formación, y hasta 8,5 veces más que un/a nigeriana.

En España (Oxfam: 2017: 1) en el año 2016 hubo 7.000 nuevas personas millonarias y la fortuna de tan sólo 3 personas equivalía a la riqueza del $30 \%$ más pobre del país, es decir, a la de 14,2 millones de personas; a su vez, mientras que en el año 2015 el 30\% más pobre vio reducida su riqueza en más de una tercera parte $(-33,4 \%)$, la fortuna de las tres personas más ricas de España aumentó un 3\%. Según señala Luque Víctor (2015: 111), durante el período 1981-2012, se ha producido un aumento de la concentración de los ingresos en los segmentos más ricos de la población española, concretamente en el $1 \%$ con más riqueza. Pero el incremento más significativo lo encontramos en el 0,01\% que más ingresa de la población, el cual multiplicó por más de cuatro veces su peso en el conjunto nacional entre los ańos 1981 y 2006. Ello contrasta con que en España la población encuestada crea que la diferencia entre lo que cobra el directivo mejor pagado de las empresas espańolas y el empleado medio es de 8,5 a 1, mientras que piensa que lo ideal sería que fuese sólo de 3 a 1 . La realidad, cuando se hizo el estudio, es que las diferencias eran de 127 a $1 .{ }^{13}$

A su vez, el Informe AROPE 2018 (EAPN, 2018: 9-12), indica que la renta media por persona completó tres ańos consecutivos de crecimiento hasta llegar a los $11.074 €$ en el año 2017, cifra que es $366 €$ más elevada que la registrada el año anterior. Este incremento, que sitúa la renta media en niveles supe-

13 La situación todavía es mucho más acentuada en Estados Unidos, pues la diferencia entre lo que creía la población encuestada que cobraba el directivo mejor pagado y el empleado medio era de 30 a 1, mientras que pensaban que lo ideal era que fuese de 7 a 1 . La realidad, cuando se hizo el estudio, mostró que las diferencias eran de 354 a 1 (Kiatpongsan, Sorapop et al, 2014: 587 a 593). 
riores a los registrados en el año 2008, pero inferiores todavía a los registrados en el 2009 es, en sí mismo, una buena noticia. Sin embargo, oculta resultados muy diferentes para importantes grupos de población y, si se tiene en cuenta el índice de precios al consumo, en lo sucesivo IPC, los datos muestran que la capacidad adquisitiva real de todos los grupos estudiados se ha reducido con respecto al año 2009. Así, por ejemplo, las personas de 65 años o más han perdido un $3,8 \%$ de renta media; las mujeres, un $10,9 \%$; las personas ocupadas, un 13,2\%; los hombres, un 13,4\%; los residentes en zonas rurales, un $14,7 \%$ y las personas en desempleo, un 25,9\%. Entre los años 2008 y 2017, la renta del $10 \%$ más rico de la población ha pasado de multiplicar por 9,6 a la renta del $10 \%$ más pobre a hacerlo por 12,93 . Ello lo confirma el Índice de Gini ${ }^{14}$, donde a pesar del descenso que se viene produciendo en Espańa desde 2014, se mantiene en 34,1; este valor es 1,7 puntos superior al registrado en 2008, y uno de los más altos de la Unión Europea: 3,7 puntos por encima de la media y sólo superado por Bulgaria, Lituania y Rumanía (datos de 2016, los últimos publicados para todos los países). Se asumen sueldos multimillonarios de algunas personas, como miembros de consejos directivos, o de algunos deportistas y artistas de élite y ello supone un gran ataque a la democracia, ya que estas personas acumulan gran poder e influencia en los medios de comunicación y en las instituciones. La soberanía del pueblo no puede quedarse en una votación formal, sino que debe llegar a las decisiones sobre la economía. Lo mismo que el monopolio está prohibido en el comercio, igualmente un poder en pocas manos debe ser prohibido.

La tasa de riesgo de pobreza de las personas jubiladas alcanzó en España en el año 2017 el 13,1\%, con lo cual ha mantenido su línea ascendente, inaugurada en el año 2014. Es importante destacar el significativo efecto que tiene el importe de las pensiones en la generación de pobreza. Así, aproximadamente 2.835.000 pensiones, el 29,6\% de las que se distribuyen en España, están por debajo del umbral de pobreza, es decir, son inferiores a $609 €$ mensuales. Concretamente lo están el 21,2\% de las pensiones de jubilación, el 42,3\% de las de viudedad y el $80,4 \%$ de las de orfandad. Por otra parte, cabe destacar que algo más de 1,5 millones de pensiones se encuentran en el tramo inmediatamente superior al límite del umbral de pobreza, es decir, su importe mensual está entre $609 €$ y $650 €$. En otras palabras, otro $15 \%$ de pensionistas está a punto de caer en la pobreza.

A nivel de Euskadi, la Encuesta de Pobreza y Desigualdades Sociales de la CAV de 2016 señalaba que 59.497 personas, equivalente al 30,7\% del total en

${ }^{14}$ El índice de Gini es un número entre 0 y 100, en donde 0 se corresponde con la perfecta igualdad, es decir, que todas las personas tienen los mismos ingresos y donde el valor 100 se corresponde con la perfecta desigualdad, lo que significa que una persona tiene todos los ingresos y las demás ninguno. 
riesgo de pobreza, no fueron atendidas por el sistema de rentas mínimas ${ }^{15}$ y que un $9 \%$ de las niñas y niños en el País Vasco viven en situación de pobreza extrema (Marcos Liliana y Ubrich Thomas, 2017: 16).

Y este panorama tiene gravísimas consecuencias, pues la desigualdad está en la base de la depresión, del síndrome de desgaste profesional, de la drogadicción, del fracaso escolar, de la obesidad, de las infancias infelices, de la participación electoral o de la desconfianza social y política. Las consecuencias psicosociales de la desigualdad son tan grandes que las personas que viven en sociedades desiguales pasan más tiempo preocupadas por cómo las ven los demás, lo que socava la calidad de sus relaciones, y el estrés resultante es factor determinante de enfermedades y problemas de salud crónicos. Además, la desigualdad está muy relacionada con la falta de igualdad de oportunidades, aumentando el riesgo de pobreza, de exclusión y la adopción de decisiones desacertadas $^{16}$. Incluso las personas ricas sufren cuando la desigualdad es demasiado grande, pues son más proclives a la depresión, la desconfianza y muchas otras dificultades sociales. El mismo Fondo Monetario Internacional admite que un exceso de desigualdad inhibe el crecimiento económico ${ }^{17}$. Por tanto, a partir de un cierto nivel económico la igualdad es un indicador más preciso para conocer el bienestar.

\section{Nuevas tecnologías y trabajo ${ }^{18}$}

Quiero fijarme especialmente en el efecto que la robótica y, en general, las nuevas tecnologías tienen en el número de personas empleadas. A modo

15 Nos referimos a tres clases de ayudas no contributivas que rigen en la Comunidad Autónoma del País Vasco: la renta de garantía de ingresos (RGI), la prestación complementaria de vivienda (PCV) y las ayudas de emergencia social (AES).

16 Sendhil, Mullainathan y Eldar, Shafir (2013), señalan que la escasez afecta a la mente y las personas actúan de manera diferente cuando perciben que algo escasea. Esas personas son hábiles gestionando los problemas a corto plazo, pero lo contrario en los problemas a largo plazo, ya que esa perspectiva desaparece perdiendo la capacidad de centrarse en otras cosas también importantes. No son ineptas, pero tienen afectado el ancho de su banda mental, de forma que el autocontrol se percibe como un reto. Estás distraída/o y te desconcentras fácilmente y eso ocurre cada día. La pobreza reduce el coeficiente intelectual entre 13 y 14 puntos, lo que es comparable a dejar de dormir una noche o a las consecuencias del alcoholismo. En definitiva, favorece que una persona se equivoque en sus decisiones.

17 Ostry, Jonathan D. et al (2014)

18 Empleo y trabajo no son lo mismo. Raventós, Daniel y Wark, Julie (2016) definen el trabajo como el «conjunto de actividades, remuneradas o no, cuyos resultados proporcionan bienes y servicios a los miembros de nuestra especie y sirven para su reproducción», mientras que el empleo se reduce a las actividades que reciben contraprestación económica. Por tanto, la diferencia fundamental entre trabajo y empleo es la gran separación entre la esfera pública o formal, donde se sitúa el trabajo productivo, y la esfera privada o informal, donde se sitúa el trabajo reproductivo, al que se refieren 
de ejemplo, en el año 1964 las 4 mayores empresas de Estados Unidos tenían un promedio de unos 430.00 personas trabajando, mientras que en el año 2011 solo daban trabajo a una cuarta parte de ellas, aunque cada empresa valía el doble; o mientras Kodak, empresa inventora de la cámara digital, a finales del año 1980 tenía 145.000 personas trabajando, en el año 2012 se declaró en bancarrota, a la vez que Instagram se vendió a Facebook por 1.000 millones de dólares. Cada vez se necesitan menos personas para tener un negocio de éxito, lo que significa que se beneficia menos gente. Cualquier producto se elabora con piezas fabricadas en distintos países del mundo, y los nuevos avances tecnológicos significan despidos masivos de trabajadores. Los académicos de la Universidad de Oxford Frey, Carl Benedikt y Osborne, Michael A. (2013), vaticinan que en 20 años el $47 \%$ de todas y todos los empleados en Estados Unidos y el 54\% en Europa correrán un alto riesgo de ser usurpados por máquinas ${ }^{19}$. Aunque en la historia siempre se han creado de manera natural nuevos trabajos que sustituyen a los desaparecidos, esta tendencia ha cambiado a partir del año 2000, debido a que la productividad está en niveles de récord avanzado la innovación más rápido que nunca, mientras que los salarios medios descienden y tenemos menos empleos. Algunos empleos resisten en la base de la pirámide, como supermercados, cadenas de comida rápida y residencias de ancianos, pero seguramente será cuestión de tiempo hasta que también desaparezcan.

Hoy existe una polarización del mercado laboral, ampliándose la brecha entre trabajos basura y trabajos fantásticos. La actual deslocalización es un paso intermedio hasta que los talleres de los países más pobres terminen también por automatizarse. Sabemos que los robots no enferman, ni se toman días libres y nunca se quejan, pero obligan a multitud de personas a emplearse en trabajos muy mal pagados y sin futuro, lo que Standing, Guy (2013) vaticina como el surgimiento de un nuevo y peligroso precariado, como clase social con salario bajo, trabajos temporales y ninguna voz en la política.

El Foro Económico Mundial (2016) afirma que la cuarta revolución industrial ya está aquí y que, como no espabilemos, su impacto social va a ser mayor que el de todas las anteriores; concretamente, pone cifras a las consecuencias que la robótica y las nuevas tecnologías van a tener en el empleo, dado que a escala mundial se destruirán 5,1 millones de puestos de trabajo netos entre 2015 y 2020. A su vez, la Comisión Europea (2016), tras el dictamen del Comité Económico y Social Europeo, concluye que, si bien la di-

con gran claridad, precisión y concisión Carrasquer, Pilar et al. (1998), destacando que la incorporación de la mujer al mundo laboral supuso toda una revolución social en el proceso de emancipación femenina, pero que todavía lejos de ser completa, se ha caído en una nueva trampa: «la doble presencia» o la doble jornada de trabajo que realizan las mujeres trabajadoras en la empresa y en el hogar.

19 El cálculo correspondiente a Europa lo realiza Bowles, Jeremy (2014). 
gitalización producirá ganancias de productividad, no se conoce con precisión su efecto sobre los niveles de empleo, aunque está claro que la tendencia futura será negativa para el mercado laboral y la organización del trabajo ${ }^{20}$. En el contexto español las consultoras Adecco y Opinno (2016), a través de una encuesta a personas expertas en recursos humanos, afirman que en el futuro las consecuencias más importantes sobre el empleo en España derivarán de los avances tecnológicos, con 4,8 puntos en una escala de 5 y que, de entre esos avances tecnológicos, la robotización del trabajo tendrá un efecto sustantivo (3,85 puntos en una escala de 5). En el caso del País Vasco el Observatorio Futurelan de Lanbide, dependiente del Gobierno Vasco, estima que para el año 2030, comparando con el empleo registrado en el año 2015, habrá un incremento en 107.454 ocupaciones, equivalente al $12 \%$, si bien con una variación considerable entre unas y otras ${ }^{21}$.

\section{Necesidad de un reparto del trabajo}

Pero un factor fundamental para valorar el empleo es el de número de horas de trabajo a la semana o al año. Precisamente la lucha por una jornada laboral que permita trabajar para vivir y no vivir para trabajar es indisociable del movimiento obrero (Elías Ángel y Rincón Leire, 2017: 48 a 50), como lo acredita la celebración anual del $10^{\circ}$ de mayo como el Día Internacional de las y los Trabajadores a causa de la Matanza de Chicago de 1886. En el siglo xix, este objetivo determinó gran parte de las reivindicaciones obreras. El Tratado de Versalles, que puso fin a la Primera Guerra Mundial, recomendaba la jornada de 8 horas diarias, las 48 horas semanales y que los domingos fuesen festivos, propuestas que fueron recogidas por la Organización Internacional del Trabajo (OIT) en 1919, con el añadido de que se librase la tarde de los sábados. En España fue el Decreto de 3 de abril de 1919 el que aprobó la jornada de 8 horas diarias y 48 horas a la semana. Desde el año 1960 la jornada laboral está fijada en 8 horas diarias y 40 horas a la semana, lo que contrasta gravemente con que en este mismo periodo se haya producido un enorme incremento de la producción y de la productividad.

Es digno de resaltar el caso de Francia con las Leyes Aubry (1998-2000) y, especialmente, la obligación de reducción de jornada de trabajo a 35 horas semanales en todas las empresas del sector mercantil de más de 20 asalariados a

${ }^{20}$ La trascendencia del asunto ha hecho que la Unión Europea en las convocatorias para el Programa del Reto Social 6 de Horizon 2020 (periodo 2018-2020), «Europa en un mundo cambiante: sociedades inclusivas, innovadoras y reflexivas", incluya, dentro de la convocatoria "Transformaciones socioeconómicas de la cuarta revolución industrial», un área de estudio referida a "La investigación para el crecimiento inclusivo: los efectos socioeconómicos de las transformaciones tecnológicas».

${ }^{21}$ Véase cuadro completo en http://futurelan.eus/es/ocupaciones2 
partir del 1 de enero de 2000, cuyos resultados corroboró un informe de la Comisión de Investigación de la Asamblea Nacional Francesa de 2014: entre 1999 y 2000, periodo en que se aplicó la Ley a grandes empresas y al sector público, se crearon 350.000 empleos directos gracias a esta medida (De la Fuente, Mikel y Zubiri, Jon Bernat, 2016: 102) 22 .

Como señala Bregman, Rutger (2016: 119 a 139) no se ha cumplido la profecía de keynes, a pesar de que ya en el año 2000 países como Francia, Países Bajos y Estados Unidos eran 5 veces más ricos que en 1930. La realidad es que se trabaja más en favor del consumismo, pero también realizando trabajos que en muchos casos son poco útiles o incluso absurdos ${ }^{23}$. La revolución feminista, con el ingreso masivo de la mujer, ha sido un factor importante de cambio en el mercado laboral. Si en el año 1970 las mujeres contribuían a los ingresos familiares entre el 2\% y el 6\%, en el año 2010 lo hacían en más del $40 \%$. Y si se incluye el trabajo no remunerado las mujeres en Europa y Estados Unidos trabajan más que los hombres. En los Países Bajos se acusa cada vez más el peso del trabajo, las horas extraordinarias, el cuidado de los hijos y la educación: en 1985, estas actividades ocupaban 43,6 horas semanales y en el ańo 2005 48,6 horas semanales. Un $75 \%$ de personas holandesas se sienten desbordadas por la falta de tiempo, una cuarta parte hace horas extraordinarias habitualmente y una de cada 8 muestran síntomas de agotamiento. Sin embargo, trabajar menos mejora el estrés, el cambio climático, la siniestralidad, el desempleo, la emancipación de la mujer, el envejecimiento de la población y también las desigualdades. El verdadero ocio, no es ni lujo ni vicio. Es tan vital para nuestros cerebros como la vitamina $\mathrm{C}$ para nuestros cuerpos. Pero para nadar en un mar de tiempo libre es preciso que la educación nos prepare no solo para integrarnos en el mercado laboral, sino también para la vida; sin embargo, muchas veces está más preocupada en la metodología y en la didáctica que en los contenidos y valores, dando capacidad para resolver problemas, pero sin preguntarse por los problemas que necesitan solución; dando conocimientos y actitudes para reali-

22 Valoran estos autores positiva esta experiencia, considerada una referencia en la conquista de mayores cotas de productividad «en especie» vía la RTT [reducción de tiempos de trabajo]. Estando cualquier experiencia plagada de contradicciones, podemos afirmar que las Leyes Aubry dan buena fe de la vigencia histórica de las políticas de RTT a favor de los trabajadores y de un reparto más justo de los excedentes productivos y sus mejoras técnicas y organizativas permanentes. Asimismo, se considera que la reducción generalizada, sustantiva, equitativa y redistributiva del tiempo de trabajo [...] es la única vía creíble para afrontar la ineludible reorganización de los trabajos reproductivos y de cuidados, en la línea de su reparto consistente entre hombres y mujeres.

23 Hay muchos trabajos que no crean riqueza, sino que solo la mueven, aunque se lleven los mejores salarios, como acontece en el caso de la banca, cuyos productos financieros les permite concentrar la riqueza en muy pocas manos. Pero concentrar la riqueza no significa crearla, ya que la banca distribuye la riqueza en su propio beneficio. Curiosamente, cuanto más eficiente es la producción menos empleo crea y menor peso ocupa en la economía, como pasa con el sector agrícola, cuya producción ha aumentado muchísimo, pero el precio de los productos se ha abaratado. 
zar un trabajo en el futuro, pero sin plantearse qué conocimientos y aptitudes queremos que tengan nuestras hijas e hijos en el futuro. En vez de preguntarnos sobre qué debemos hacer para ganarnos la vida, debiéramos preguntarnos por cómo deseamos ganarnos la vida. Por tanto, hay que reestructurar la educación en función de los nuevos ideales, incorporando contenidos en filosofía, historia y arte, de manera que tengan un papel relevante. Hay que superar el dogma de que todo trabajo es relevante, y también negar que un salario más alto refleja automáticamente un trabajo de mayor valor social Bregman, Rutger (2016: 140 y 141). Solo si nos tomamos el tiempo necesario seremos capaces de gestionar la buena vida y, afortunadamente, diversas encuestas en varios países demuestran que sí estamos dispuestos a cambiar parte del poder adquisitivo por más tiempo libre (Skidelsky, Robert y Edward, 2012: 29-30).

\section{Una nueva política de reparto}

Para ello es importante reflexionar sobre cómo las riquezas acumuladas y los beneficios privados han sido logrados con el esfuerzo de todas las personas. Mazzucato, Mariana (2014) nos explica cómo la innovación tecnológica, en lo que se refiere a la creación de internet, el sistema GPS, las pantallas táctiles, los dispositivos de grabación, los buscadores de algoritmos o los distintos tipos de baterías, entre otros muchos, ha sido el resultado de una financiación gubernamental a lo largo de varias décadas, es decir, de un esfuerzo colectivo por parte de la sociedad, a través de sus impuestos. Sin embargo, el resultado de la socialización de los costes ha derivado en la privatización de los beneficios, tal como ocurre en la innovación tecnológica, pero también en el sector bancario y financiero, así como en la crisis económica más reciente, en la cual las personas ricas se han hecho más ricas, pero los costes se han facturado a la sociedad.

En teoría, dicha financiación se devuelve al Gobierno y a la comunidad mediante los impuestos corporativos, pero en la práctica sabemos que muchas veces no es así, pues los paraísos fiscales, la evasión de impuestos, así como la bajada de impuestos a empresas lo evitan, pues hay un sistema tributario que no grava suficientemente a quienes más tienen, siendo muy tolerante con el fraude y los paraísos fiscales ${ }^{24}$. Éste es un fenómeno que va en aumento, ya que en la última década la recaudación de impuestos a sociedades ha disminuido casi un 50\% (Oxfam-Intermon, 2016: 13). Además, el peso fiscal ha recaído

${ }^{24}$ La filtración de los Papeles de Panamá puso en la palestra el asunto de los paraísos fiscales. Según la Red para la Justicia Global (Turner George, 2016), en 2015 había unos 26 billones de euros (32 trillones de dólares) ocultos y libres de impuestos en los diversos paraísos fiscales, lo que supone un tercio del PIB mundial, que asciende a 75 billones. 
de manera creciente sobre el consumo y el trabajo, en vez de sobre las transacciones financieras. En 1970 en Estados Unidos las acciones se mantenían en cartera un promedio de 5 años, mientras que en el año 2010 el promedio era de 5 días. Un impuesto por cada transacción evitaría que los operadores financieros se beneficiasen de la compraventa de valores financieros en una fracción de segundo.

También creo de sumo interés, como hace Raventós Daniel (2018) plantear una renta máxima donde a partir de determinada cantidad no se puede ganar más, es decir, $100 \%$ de tasa impositiva. Ello no solo da más dinero para repartir y posibilitar las prestaciones públicas, sino que al mismo tiempo, al evitar las grandes fortunas, refuerza la libertad de la gran mayoría.

\section{Derecho a la renta básica universal e incondicional}

La renta básica puede ser un instrumento muy importante en la redistribución de la riqueza, permitiendo acabar con la pobreza y garantizar la existencia material de cada persona y su libertad. Según el concepto aprobado por la Basic Income Earth Network, que es la red internacional que trabaja a favor de la implementación de la renta básica, ésta se traduce en un pago periódico, individual e intransferible. Es universal e incondicional, lo que significa que se distribuye a toda la población, independientemente de la edad, la raza, el género, el origen, o la condición socioeconómica y laboral. No está atada a ningún estatus económico o laboral, ni a un determinado nivel de renta y permite una fuerte incidencia en las áreas del trabajo, de la igualdad de género, de la democracia y la participación ciudadana, de la juventud y de la eficiencia de la administración (Elías Ángel y Rincón Leire, 2016).

Como dice Bregman, Rutger (2016: 37 a 39), frente a determinados prejuicios existentes, hay que subrayar que la renta básica no es fútil, ya que implica la erradicación de pobreza, y su naturaleza universal permite que la ciudadanía lo respalde mucho más. Tampoco es peligrosa, ya que la gente tendemos a trabajar, y al ser una ayuda incondicional no desmotiva a la hora de conseguir ingresos por el trabajo. Lejos de ser perversa evita la humillación que genera el actual sistema de control social, y permite una mejor redistribución de la riqueza, así como una más limitada intervención gubernamental. Además, desde la experiencia del año 1973 en Mincome ${ }^{25}$ y el reparto de dividendos que desde

25 Es una pequeńa población de 13.000 habitantes de Dauphin, al noroeste de Winnipeg, en Canadá. Resulta llamativo que no se hubiera descubierto hasta que la profesora Forget, Evelyn (2013), de la Universidad de Manitoba, rescató sus documentos en el ańo 2009 en el Archivo Nacional de Canadá. Esta experiencia consistió en que 1.000 familias, alrededor del 30\% de la población de Dauphin, cobraba de manera incondicional según el número de miembros que, por 
hace cinco décadas existe en Alaska, se han multiplicado experiencias en todo el mundo que avalan su éxito ${ }^{26}$.

La situación es favorable a que tengamos una renta básica universal e incondicional, pues la mayor flexibilidad en el puesto de trabajo exige que dispongamos también de una mayor seguridad. De otra parte, si el mantenimiento del empleo exige una jornada más reducida que permita el reparto del mismo, la renta básica se convierte en un ingreso necesario para complementar el salario y garantizar a cada persona unos recursos económicos adecuados.

ejemplo, en caso de ser cuatro equivalía a 19.000 \$ al año. Funcionó durante 4 años, pero el cambio derivado de la llegada de los conservadores al poder terminó con este programa y se archivaron más de 2.000 cajas de documentos, sin haber analizados los resultados. Sin embargo, como ha acreditado Forget, Evelyn, éstos fueron de un éxito clamoroso, permitiendo que la tasa de natalidad disminuyese, el rendimiento escolar aumentarse, y la salud mejorase, reduciéndose las hospitalizaciones hasta en un 8,5\%, y disminuyendo la violencia doméstica, así como los trastornos mentales.

26 Bregman, Rutger (2016: 28 a 31) nos cuenta cómo los pobres son quienes mejor gestionan el dinero para hacer frente a sus necesidades, pues, la pobreza es sobre todo falta de dinero y no la estupidez de quienes la sufren. Un estudio del Banco Mundial demuestra que en el $82 \%$ de los casos donde se ha dado dinero en países de África, Asia y América no se ha utilizado para consumo de tabaco o de alcohol, que se han reducido grandemente. Por ejemplo, en una zona empobrecida del oeste de Kenia, la organización Givedirectly ha demostrado que los ingresos fueron estimulados de forma duradera hasta un 38\%, se potenció la adquisición de viviendas y de ganado hasta un $58 \%$, y se redujo en un $42 \%$ los días que los nińos pasaban hambre; además, el $93 \%$ de cada donación se entregó en mano a los receptores. También en 2008 el gobierno de Uganda distribuyó 400 \$ a 12000 personas de entre 16 y 35 ańos con la exigencia de que presentaran un plan de negocio. 5 años más tarde los efectos fueron muy positivos habiendo invertido en su propia educación e iniciativas de negocios, que permitió que los ingresos ascendieran en un $50 \%$ y las posibilidades de ser contratados se habían incrementado en más de un $60 \%$. Otro programa ugandés distribuyó en el norte del país 150 \$ a más de 1800 mujeres, que pudieron duplicar los ingresos. Múltiples experiencias acreditan una correlación entre el desembolso económico incondicional y la reducción de la delincuencia, la mortalidad infantil, la desnutrición, el embarazo adolescente, el absentismo escolar, el rendimiento académico, el crecimiento económico y la igualdad entre sexos. Así, en Namibia la desnutrición bajó del $42 \%$ al $10 \%$, el absentismo escolar del $40 \%$ al $0 \%$, y la delincuencia se redujo en un $42 \%$. En Malaui la asistencia de niñas y mujeres a la escuela aumentó en un 40\%. En 2010 las ayudas incondicionales llegaron a 110 millones de familias en 45 países del Sur. Investigadores de Manchester resumen los cuatro grandes beneficios de estos programas: primero, las familias dan un buen uso al dinero; segundo, la pobreza se reduce; tercero se producen diversos beneficios de larga duración en ingresos, salud e impuestos; y cuarto estos programas son menos costosos que las alternativas. Además, dando el dinero directamente a los beneficiarios se eliminan las corrupciones en el funcionariado, y se estimula la economía y el empleo. Algo parecido nos enseńó Lloyd, Pendleton, director del grupo de asistencia a las personas sin techo de Utah, quien en 2005 realizó un experimento con 17 personas indigentes muy recalcitrantes a quienes les dio apartamentos gratis y en 2 años, gracias al absoluto éxito, amplió el programa progresivamente. Además, resultó más barato, ya que un vagabundo en la calle costaba al año 16.670 \$ mientras que un apartamento y orientación profesional se quedaba en los $11.000 \$$. Esto ha dado la vuelta al mundo y también se ha incorporado recientemente en la estrategia del Gobierno Vasco. 


\section{Propuesta de creación de un Observatorio para el reparto justo de la riqueza}

Abogo por la creación de un Observatorio para el reparto justo de la riqueza, que permita dar visibilidad a la riqueza existente y a las personas que la poseen, contrarrestando esa imagen falsa e interesada de que no hay riqueza suficiente para financiar un buen sistema de protección social, que incluya derechos universales como la renta básica. En este sentido Oxfam (2018: 21) en su publicación Premiar el trabajo, no la riqueza, entre las cinco recomendaciones, que en la pág. 21 realiza a los Gobiernos sobre desigualdad, está la siguiente:

Colaborar para lograr una revolución de datos sobre desigualdad. Todos los países deberían aspirar a producir datos anuales sobre la riqueza y los ingresos de todos sus habitantes, especialmente los correspondientes al $10 \%$ y al $1 \%$ más rico. Además de financiar más encuestas de hogares, deben publicarse otras fuentes de datos que arrojen luz sobre la concentración de ingresos y riqueza en lo más alto.

Y en nota a pie de página añade:

Esto debería incluir datos sobre ingresos, patrimonio e impuestos; datos sobre mercados de patrimonio y bienes de lujo; datos de las empresas que gestionan la riqueza y fortunas; y estudios sobre salarios. Estos datos deberían desagregarse por género, edad, ocupación, región y, si corresponde, grupos étnicos.»

Y ello, porque como dijo Piketty, Thomas (2013: 36), no hay que caer en determinismos económicos, pues

La historia de la distribución de la riqueza es siempre profundamente política y no podría resumirse en mecanismos puramente económicos (...) La historia de las desigualdades depende de las representaciones que nos hacen los actores económicos, políticos y sociales, de lo que es justo y de lo que no lo es, de las relaciones de fuerza entre esos actores y de las elecciones colectivas que resultan de ello; es el producto conjunto de todos los actores interesados. (...) El resultado de esta historia dependerá de las representaciones que se hacen las diferentes sociedades de las desigualdades, y de las políticas e instituciones que se atribuyen para modelarlas y transformarlas, en uno u otro sentido.

De ahí la importancia de un Observatorio para el reparto justo de la riqueza, que desde la Universidad pública y con el apoyo de múltiples entidades, contribuya al desarrollo de políticas que permitan un reparto justo de la riqueza y evite nuevos casos de disonancia cognitiva, como la que siguió a la quiebra del banco de inversión Lehman Brothers el 15 de septiembre de 2008, siendo clave el momento inmediatamente posterior a cualquier crisis para conocer si hay una apertura a nuevas ideas y alternativas, o un apuntalamiento de las viejas convicciones. 


\section{Bibliografía}

Adecco y Opinno (2016): Informe Adecco sobre el futuro del trabajo en España, Pozuelo de Alarcón, Adecco, disponible a 14 de noviembre de 2018 en https://www.ceu.es/ joblab/documentacion/informeAdecco.pdf .

Arope (2018): El estado de la pobreza. Seguimiento del indicador de pobreza y exclusión social en España: 2008-201, EAPN, disponible a 14 de noviembre de 2018 en https:// www.eapn.es/estadodepobreza/ARCHIVO/documentos/Informe_AROPE_2018.pdf

Bowles, Jeremy (2014): The computerisation of European Jobs, disponible a 14 de noviembre de 2018 en http://bruegel.org/2014/07/the-computerisation-of-european-jobs/

Branko, Milanovic (2007): "Why We All Care About Inequality (but are loath to admit it)», Challenge, vol. 50, no. 6, November/December 2007, disponible a 14 de noviembre de 2018 en https://pdfs.semanticscholar.org/7fdb/845b4d5bfd36ebf4b3c11 dd68417192felea.pdf

Bregman, Rutger (2016): Utopía para realistas, ed. Salamandra, disponible a 14 de noviembre de 2018 en https://kupdf.net/download/utopia-para-realistas-rutgerbregman_59c359ad08bbc577186870d0_pdf

Carrasquer, Pilar et al. (1998): «El trabajo reproductivo», Papers, n. ${ }^{o}$ 55, págs. 95-114, disponible a 14 de noviembre de 2018 en https://ddd.uab.cat/pub/papers/02102862 n55/02102862n55p95.pdf

Caselli, Francesco y Feyrer, James (2007): «The Marginal Product of Capital», FMI, http://personal.lse.ac.uk/casellif/papers/MPK.pdf

Comisión Europea (2016): «Dictamen del Comité Económico y Social Europeo sobre "Los efectos de la digitalización sobre el sector de los servicios y el empleo en el marco de las transformaciones industriales" (Dictamen exploratorio)», Diario Oficial de la Unión Europea, 5-1-16, 2016/C 013/24, disponible a 14 de noviembre de 2018 en https:// eur-lex.europa.eu/legal-content/ES/TXT/PDF/?uri=CELEX:52015AE0765\&from=ES

Comité Internacional de los Derechos Económicos, Sociales y Culturales (1990): Observación general N.o 3, La indole de las obligaciones de los Estados Partes (párrafo 1 del artículo 2 del Pacto), disponible a 14 de noviembre de 2018 en https:// tbinternet.ohchr.org/_layouts/treatybodyexternal/Download.aspx?symbolno=INT\%2 fCESCR $\% 2 f G E C \% 2 f 4758 \&$ Lang=en

Comité Internacional de los Derechos Económicos, Sociales y CulturaLES (1998): Observación General N. ${ }^{\circ}$ 9, La aplicación interna del Pacto, doc. ONU: E/C.12/1998/24, 3 de diciembre de 1998, párrafo 5.

Comité Internacional de los Derechos Económicos, Sociales y Culturales (2012): Observaciones Finales: España, doc. ONU: E/C.12/ESP/C0/5, 18 de mayo de 2012, párrafo 8 .

Comité Internacional de los Derechos Económicos, Sociales y Culturales (2015): Comunicación núm. 2/2014, E/C.12/55/D/2/2014, disponible a 14 de noviembre de 2018 en https://tbinternet.ohchr.org/_layouts/treatybodyexternal/ Download.aspx?symbolno=E/C.12/55/D/2/2014\&Lang=en

Coyle, Diane (2014): GDP. A Brief but affectionate history, Princeton University Press (Nueva Jersey), disponible a 14 de noviembre de 2018 en http://www.eaco.eu/wpcontent/uploads/2015/09/review_3-15.pdf

De la Fuente, Mikel et al. (2018): Hacia una nueva reforma de las pensiones? Notas para el Pacto de Toledo, disponible a 14 de noviembre de 2018 en http://documentos.fedea. net/pubs/fpp/2018/10/FPP2018-09.pdf 
De La Fuente, Mikel y Zubiri, Jon Bernat (2016): «Efectos sobre el empleo de la reducción del tiempo de trabajo: aprendizajes de la experiencia francesa», Lan Harremanak, n. ${ }^{\circ} 34$, págs. 79-105, disponible a 14 de noviembre de 2018 en http://www.ehu.eus/ ojs/index.php/Lan_Harremanak/article/view/16558/14750

Elías, Angel y Rincón, Leire (2016): "Algunas virtualidades de la renta básica», Boletín Hegoa, n. ${ }^{\circ} 48$, disponible a 14 de noviembre de 2018 en http://boletin.hegoa.ehu.es/mail/45

Elías, Ángel y Rincón, Leire (2017): Políticas que favorecen la inclusión, Zerbitzuan 64, págs. 48 a 50, disponible a 14 de noviembre de 2018 en http://www.zerbitzuan.net/ documentos/zerbitzuan/Politicas_inclusion.pdf

Firebaugh, Glenn (2009): The new geography of global income inequality. Harvard University Press.

Forget, Evelyn (2013): The Case for Basic Income in Canada, disponible a 14 de noviembre de 2018 en https://www.researchgate.net/publication/275648949_The_Case_ for_Basic_Income_in_Canada

Foro Económico Mundial (2016): The Future of Jobs. Employment, Skills and Workforce Strategy for the Fourth Industrial Revolution, Cologny, disponible a 14 de noviembre de 2018 en http://www3.weforum.org/docs/WEF_Future_of_Jobs.pdf

Frey, Carl Benedikt y Osborne, Michael A. (2013): The Future of Employment: How Susceptible Are Jobs to Computerisation, Oxford Martin School, disponible a 14 de noviembre de 2018 en https:/www.oxfordmartin.ox.ac.uk/downloads/academic/The Future_of_Employment.pdf

Grove, Chris e IKawau, Daniela (2015): «Un paso histórico hacia el acceso a la justicia por violaciones de DESC en la ONU», publicado en la web openDemocracy y disponible a 14 de noviembre de 2018 en https://www.opendemocracy.net/openglobalrights/ chris-grove-daniela-ikawa/un-paso-hist-rico-hacia-el-acceso-la-justicia-por-violaci

Kiatpongsan, Sorapop et al. (2014): How much (more) should CEOs make? A universal desire for more equal pay. Perspectives on Psychological Science, vol. 9, n. ${ }^{\circ}$ 6, noviembre de 2014, págs. 587-593, disponible a 14 de noviembre de 2018 en https://www. hbs.edu/faculty/Publication\%20Files/kiatpongsan\%20norton\%202014_f02b004ac2de-4358-9811-ea273d372af7.pdf

Kroll, Luisa y Dolan, Kerry (2018): The World's Billionaires. 2018 Ranking, Forbes, consultado el 14 de noviembre 2018 y disponible a 14 de noviembre de 2018 en https://www.forbes.com/billionaires/\#997c948251c7

Luque, Víctor (2015): "A propósito de Piketty: evolución de la desigualdad en España», disponible a 14 de noviembre de 2018 en http://eprints.ucm.es/46408/1/2015-28$1 \% 2886-115 \% 29 . p d f$

Marcos, Liliana y Ubrich, Thomas (2017): Desheredados. Desigualdad infantil, igualdad de oportunidades y políticas públicas en España. Anexo Euskadi, Save The Children España disponible a 14 de noviembre de 2018 en https://www.savethechildren. es/sites/default/files/imce/docs/save_the_children-anexo_vasco_espanaol_web.pdf

Mazzucato, Mariana (2013): The Entrepreneurial State: Debunking Public vs. Private Sector Myths, Londres, Anthem, 2013.

Ostry, Jonathan D. et al. (2014): Redistribution, Inequality, and Growth, FMI, disponible a 14 de noviembre de 2018 en https://www.imf.org/external/pubs/ft/sdn/2014/ sdn1402.pdf

Oxғам (2016): Beneficios para quién, disponible a 14 de noviembre de 2018 en https:// www.oxfamintermon.org/es/documentos/11/05/16/beneficios-para-quien-impactosocial-de-abusos-fiscales 
OXfam (2017): Una economía para el 99\%- España, disponible a 14 de noviembre de 2018 en https://www.oxfamintermon.org/sites/default/files/documentos/files/ Informe-Una-economia-para-99-espana-oxfam-intermon.pdf

Oxfam (2017): Una economía para el 99\%, disponible a 14 de noviembre de 2018 en https://www.oxfamintermon.org/sites/default/files/documentos/files/Informe-Unaeconomia-para-99-oxfam-intermon.pdf

Oxғам (2018): Premiar el trabajo, no la riqueza, disponible a 14 de noviembre de 2018 en https:/www.oxfamintermon.org/sites/default/files/documentos/files/premiar-trabajono-riqueza.pdf

Oxfam y Development Finance International (2018): Indice de compromiso con la reducción de la desigualdad (CRI) 2018, disponible a 14 de noviembre de 2018 en https://www.oxfam.org/es/informes/indice-de-compromiso-con-la-reduccion-de-ladesigualdad-cri-2018

Piketty, Thomas (2013): Capital in the twenty-first century, Belknap Press, Cambridge, disponible a 14 de noviembre de 2018 en https://dowbor.org/blog/wp-content/ uploads/2014/06/14Thomas-Piketty.pdf

Raventós, Daniel y WARK, Julie (2016): «Trabajo remunerado, trabajo doméstico, trabajo voluntario y Renta Básica», Sin Permiso, 8-5-2016, disponible a 14 de noviembre de 2018 en http://www.sinpermiso.info/textos/trabajo-remunerado-trabajodomestico-trabajo-voluntario-y-renta-basica

Raventós, Daniel (2018): «Renta básica y renta máxima», Sin Permiso, 27-5-2018, disponible a 14 de noviembre de 2018 en http://www.sinpermiso.info/textos/renta-basicay-renta-maxima

SEndHIL, Mullainathan y Eldar, Shafir (2013): Scarcity: why having too little means so much, Editorial Reviews

SEYfFerth, Ansgar (2014): «La omnipresente media estadística - ¿Qué nos dice y qué nos oculta?», disponible a 14 de noviembre de 2018 en https://www.huffingtonpost.es/ ansgar-seyfferth/la-omnipresente-media-estadistica_b_5481974.html

Seyfferth, Ansgar (2014), “¿Cómo se mide la desigualdad?», disponible a 14 de noviembre de 2018 en https://es.weforum.org/agenda/2014/11/como-se-mide-la-desigual$\mathrm{dad} /$

Skidelsky, Robert y Edward (2012); How Much is Enough? The Love of Money and the Case for the Good Life, Nueva York, Penguin.

Standing, Guy (2013): El Precariado. Una nueva clase social, Barcelona, Ed. Pasado y Presente.

Turner, George (2016): Review of 2016, Tax Justice Network, disponible a 14 de noviembre de 2018 en https://www.taxjustice.net/2016/12/31/review-of-2016/

Walmsley, Terrie L. et al. (2007): Measuring the Impact of the Movement of Labour Using a Model of Bilateral Migration Flows, Banco Mundial, disponible a 14 de noviembre de 2018 en https://www.gtap.agecon.purdue.edu/resources/download/2398.pdf .

Wilkinson, R. G. et al. (2011): The spirit level: Why Greater Equality Makes Societies Stronger, Bloomsbury Publishing USA, Nueva York, disponible a 14 de noviembre de 2018 en https:/www.taxjustice.net/2016/12/31/review-of-2016/ http:// emilkirkegaard.dk/en/wp-content/uploads/The-Spirit-Level-Why-Greater-EqualityMakes-Societies-Stronger-Kate-Pickett-400p_1608193411.pdf 